\title{
How Can We Predict the Successful Outcome After Treatment in Achalasia Patients? (Gastroenterology 2012;143:328-335)
}

\section{Su Jin Hong}

Department of Internal Medicine, Soonchunhyang University College of Medicine, Bucheon, Gyeonggi-do, Korea

\section{Summary}

Typical symptoms of achalasia are dysphagia, regurgitation of undigested food, chest pain, weight loss and respiratory complications. In previous studies, esophageal manometry has been proposed as a useful test to determine whether patients should receive re-treatment. Decreased lower esophageal sphincter (LES) pressure to less than $10 \mathrm{mmHg}$ has been reported to be a good predictor of long-term treatment success. ${ }^{1,2}$ However, some achalasia patients with persistent symptoms after treatment had low or absent LES pressure. ${ }^{3,4}$ On the other hand, esophagogastric junction (EGJ) compliance is an important pathophysiological factor in the cases of achalasia and gastroesophageal reflux disease (GERD). A commercial measurement device, EndoFLIP system (endoscopic functional luminal imaging probe; Crospon, Galway, Ireland) was recently introduced to test compliance or distensibility of EGJ. The EndoFLIP uses impedance planimetry to determine multiple adjacent cross sectional areas (CSAs) within a cylindrical bag placed in a tubular organ during volumetric distention. The additional measure of the corresponding intrabag pressure allows assessment of the CSA pressure re- sponse (distensibility) of the distended area. Rohof et al ${ }^{5}$ performed the study to compare the EGJ distensibility and LES pressure as a better and more integrated parameter after treatment in the patients with achalasia.

This study was performed in 15 healthy volunteers and 34 patients with achalasia. Healty volunteers underwent EndoFLIP measurement. Achalasia patients underwent esophageal manometry, EndoFLIP measurement of the EGJ, and timed barium esophagography. The symptoms were assessed using the Eckardt score. In 4 patients, the EndoFLIP probe could not pass the EGJ. Remained 30 achalasia patients could take the EndoFLIP test to estimate distensibility. Seven of the 30 patients were newly diagnosed and were measured before and 3 months after treatment. The authors included pneumodilation and laparoscopic Heller myotomy as the treatment options.

The authors defined successful treatment as an Eckardt symptom score of $\leq 3$. The 30 achalasia patients were divided into the successfully treated group (18 patients) and the treatment failure group (12 patients) by the Eckardt symptom score. Healthy volunteers had a mean EGJ distensibility of $6.3 \pm 0.7$ $\mathrm{mm}^{2} / \mathrm{mmHg}$ using a $50 \mathrm{~mL}$ volume distention. Untreated patients $\left(0.7 \pm 0.9 \mathrm{~mm}^{2} / \mathrm{mmHg} ; P<0.001\right)$ had a significantly

Received: September 1, 2012 Revised: September 26, 2012 Accepted: September 27, 2012

(c) This is an Open Access article distributed under the terms of the Creative Commons Attribution Non-Commercial License (http://creativecommons. org/licenses/by-nc/3.0) which permits unrestricted non-commercial use, distribution, and reproduction in any medium, provided the original work is properly cited.

*Correspondence: Su Jin Hong, MD

Department of Internal Medicine, Soonchunhyang University College of Medicine, Jomaru-ro 170, Wonmi-gu, Bucheon, Gyeonggi-do 420-767, Korea

Financial support: None.

Tel: +82-32-621-5087, Fax: +82-32-621-5080, E-mail: sjhong@schmc.ac.kr

Conflicts of interest: None. 
lower EGJ distensibility compared with healthy volunteers. The EGJ distensibility of both unsuccessfully treated and successfully treated patients was lower than that of healthy volunteers in a 50 $\mathrm{mL}$ volume distention $\left(1.6 \pm 0.3 \mathrm{~mm}^{2} / \mathrm{mmHg} ; P<0.01\right.$ and $4.4 \pm 0.5 \mathrm{~mm}^{2} / \mathrm{mmHg} ; P=0.02$, respectively). In patients with achalasia, EGJ distensibility correlated with esophageal emptying ( $\mathrm{r}=-0.72 ; P<0.01)$ and symptoms $(\mathrm{r}=0.61 ; P<0.01)$ and was significantly increased with treatment. The patients with impaired distensibility and low LES pressure showed significantly more stasis on their timed barium esophagogram compared to the patients with normal distensibility and low LES pressure. The authors concluded EGJ distensibility is a better parameter than LES pressure for evaluating efficacy of treatment for achalasia.

\section{Comment}

EGJ distensibility was estimated by a commercially developed EndoFLIP system which uses impedance planimetry to determine multiple adjacent CSAs with a $25 \mathrm{~mm}$ cylindrical balloon probe housing an array of 16 pairs of electrodes spaced $5 \mathrm{~mm}$ apart. EndoFLIP has been used to evaluate the EGJ in GERD, eosinophilic esophagitis, and during the operation for functional esophageal disease. $^{6-9}$

In GERD, increased EGJ compliance is a key pathophysiological abnormality leading to increased quantity of reflux. A previous study showed 2-3 folds of increase in EGJ distensibility in GERD group compared with control group, particularly at 20-30 mL distention volumes. ${ }^{6}$ Such EGJ distensibility measured by EndoFLIP revealed poor correlation with EGJ distensibility by endoscopic examination (flap valve grade). The reflux volumes are attributable to EGJ distensibility. The GERD patients who have increased distensibility and large reflux volumes may benefit from anatomic correction, by either surgical or endoluminal treatments. On the other hand, the GERD patients with normal EGJ distensibility may have little gain from such interventions.

Esophageal distensibility was significantly reduced in the patients with eosinopilic esophagitis compared to controls. ${ }^{7}$ It suggests that reduced esophageal distensibility may lead to dysphagia and impaction without mechanical obstruction in eosinophilic esohagitis. EndoFlip measurements were already tried during operation such as Heller myotomy and Nissen fundoplication. ${ }^{8,9}$

Achalasia is a neurogenic esophageal motility disorder characterized by esophageal aperistalsis and incomplete relaxation of LES following swallows. The goal of the treatment for achalasia is to decrease distal obstruction, which can be treated by botulinum toxin injection, pneumatic dilatation or surgical myotomy. Recently, peroral endoscopic myotomy has been introduced as the endoscopic treatment method in patients with achalasia. However, the clinical responses after treatment are among patients with achalasia. Pneumatic dilatation is usually considered as the first-line therapy in achalasia. Surgical myotomy has been offered to patients who do not respond to pneumatic dilatation. In general, long-term outcome of pneumatic dilatation is better in patients with age older than 40 years, female sex, large balloon usage, post-pneumatic dilatation LES pressure $<10 \mathrm{mmHg}$, post-pneumatic dilatation LES pressure reduction by $>50 \%$, and complete emptying in timed barium esophagogram. In addition to these factors, Rohof et $\mathrm{al}^{5}$ suggested that the post-treatment EGJ distensibility should be considered as a better parameter than LES pressure for estimating the efficacy of treatment for achalasia. Even if the patients have low LES pressure, EGJ distensibility can be impaired, leading to impaired esophageal emptying and recurrent symptoms. In conclusion, this study recommends the assessment of EGJ distensibility by EndoFLIP which is a valuable tool to predict the outcome after pneumatic dilatation or surgical myotomy in achalasia patients.

\section{References}

1. Eckardt VF, Aignherr C, Bernhard G. Predictors of outcome in patients with achalasia treated by pneumatic dilation. Gastroenterology 1992;103:1732-1738.

2. Hulselmans M, Vanuytsel T, Degreef T, et al. Long-term outcome of pneumatic dilation in the treatment of achalasia. Clin Gastroenterol Hepatol 2010;8:30-35.

3. Katz PO, Richter JE, Cowan R, Castell DO. Apparent complete lower esophageal sphincter relaxation in achalasia. Gastroenterology 1986;90:978-983.

4. Mearin F, Malagelada JR. Complete lower esophageal sphincter relaxation observed in some achalasia patients is functionally inadequate. Am J Physiol Gastrointest Liver Physiol 2000;278:G376G383.

5. Rohof WO, Hirsch DP, Kessing BF, Boeckxstaens GE. Efficacy of treatment for patients with achalasia depends on the distensibility of the esophagogastric junction. Gastroenterology 2012;143:328-335.

6. Kwiatek MA, Pandolfino JE, Hirano I, Kahrilas PJ. Esophagogastric junction distensibility assessed with an endoscopic functional luminal imaging probe (EndoFLIP). Gastrointest Endosc 2010;72: 272-278.

7. Kwiatek MA, Hirano I, Kahrilas PJ, Rothe J, Luger D, Pandolfino JE. Mechanical properties of the esophagus in eosinophilic esophagitis. Gastroenterology 2011;140:82-90.

8. Perretta S, Dallemagne B, Donatelli G, Diemunsch P, Marescaux J. Transoral endoscopic esophageal myotomy based on esophageal 
function testing in a survival porcine model. Gastrointest Endosc 2011;73:111-116.

9. Perretta S, Dallemagne B, McMahon B, D'Agostino J, Marescaux
J. Video. Improving functional esophageal surgery with a "smart" bougie: Endoflip. Surg Endosc 2011;25:3109. 